\title{
Person-centred care in a digital hospital: observations and perspectives from a specialist rehabilitation setting
}

Letitia Burridge, Michele Foster, Rachel Jones, Timothy Geraghty, Sridhar Atresh.

\begin{abstract}
Objective This study investigated use of electronic medical records (eMRs) in a spinal cord injury rehabilitation unit and the implications for person-centred care.

Methods This exploratory mixed methods study conducted 17.5 hours of observations of practitioner-patient encounters, 50 patient experience surveys and 10 focus groups with 53 practitioners. Descriptive statistics and qualitative analysis were integrated into key themes. Results Practitioners in this specialised setting were reconciling the emergent challenges of eMR in practice with the advantages of improved accessibility and documentation legibility. eMR increased task complexity and information retrieval, particularly for nurses. Some documentation was an uneasy fit with the specialised setting, disrupting informal communications and aspects of person-centred care.
\end{abstract}

Conclusions Technological change closely aligned with frontline practice brought expected and unexpected challenges that may resolve over time. Practitioners' persistence and adaptability demonstrated their commitment to person-centred care in the digital environment. The impact of this less visible work of professional discretion seemed to vary primarily by discipline-specific roles, with nurses experiencing the greatest pressure. 


\section{What is known about this topic?}

Integrated electronic medical records (eMRs) bring benefits but challenge person-centred care.

\section{What does this paper add?}

These first insights regarding frontline implementation of eMR in spinal injury rehabilitation suggest nursing challenges when seeking to fit specialised work into the generic eMR.

However, most patients reported receiving person-centred care.

\section{What are the implications for practitioners?}

Commitment to person-centred care appears to strengthen practitioners' perseverance with the eMR implementation challenges. 


\section{INTRODUCTION}

Health systems worldwide are exploring innovations to improve the quality, safety and efficiency of care while maintaining a strong person-centred approach ${ }^{1}$. Digital technology, especially integrated information systems, is part of the solution ${ }^{2}$. The integrated electronic medical record (eMR) or electronic health record is characteristic of such initiatives ${ }^{3}$. The eMR is lauded for improving data quality, data storage and retrieval and clinical decisionmaking ${ }^{4}$. Although early evidence regarding the benefits of using eMR is mixed across different settings ${ }^{5}$, it is generally positive with regard to improvements in quality, efficiency and provider satisfaction ${ }^{6}$. The positive impact of eMR is evident in hospitals in the $\mathrm{US}^{7}$ in effective patient monitoring over time and improved communication with patients ${ }^{8}$; and in positive experiences among professionals and patients ${ }^{9}$. Conversely, there are lingering concerns about the impact on person-centred care (PCC). The American Medical Informatics Association has acknowledged that eMR is generally not designed to facilitate PCC ${ }^{10}$. The disease framework of eMR data inhibits the representation of patient information in connected, contextualized, or narrative forms ${ }^{11}$. Varpio et al. ${ }^{12}$ found that data fragmentation and free-text restrictions in eMR compromised hospital practitioners' ability to create and understand individual paediatric patients' stories. Equally the standardisation of functions within eMR systems can restrict some aspects of specialist patient care ${ }^{4}$. Asan and colleagues $^{2}$ also found that using eMR meant less time for interacting with the patient, while

Darr et al. ${ }^{13}$ discovered that doctors hold mixed views about the benefit of eMRs if it negatively impacts on interaction with patients.

Nevertheless, practitioners are experts in negotiating the limitations of their tools in practice ${ }^{14}$ and adapting eMR interactions to offset its challenges to $\mathrm{PCC}^{15}$. The core task with information system innovations is to leverage strengths and overcome limitations ${ }^{16}$. This study describes experiences from one specialist rehabilitation setting in a tertiary hospital in 
south-east Queensland where a hospital-wide eMR system is being implemented. The study aims were to observe and investigate how rehabilitation practitioners are working with eMR in their interactions with patients, and to identify the challenges and opportunities this work generates for PCC from patient and practitioner viewpoints.

\section{METHODS}

\section{Design}

This mixed methods study incorporated three components: (1) structured observations of practitioner-patient interactions to document how eMRs were being used; (2) focus groups with practitioners to explore experiences of eMRs in practice; and (3) patient experience surveys to describe how patients valued the eMRs as part of their care.

\section{Setting}

With institutional and university ethics approval, the study was conducted within the 40-bed Spinal Injury Rehabilitation Unit based at the Princess Alexandra Hospital, Brisbane. The only such unit in Queensland, it operates on a person-centred, interdisciplinary, goal-based framework that is supported by linked services in other locations: a transitional rehabilitation program and spinal outreach team, including outpatient clinics, as part of the continuum of the Queensland Spinal Cord Injuries Service. The eMR hardware comprises Workstations on Wheels or laptop computers for direct patient interaction such as goal planning, ward rounds or medication management, and desktop or wall-mounted computers away from patient areas for documentation.

\section{Participants}

A convenience sample of practitioners was recruited with the assistance of clinical managers in each location. A convenience sample of adult patients was recruited with the assistance of 
an attending practitioner. All potential participants received a verbal and written explanation of the study, and those who agreed to participate provided voluntary informed consent in writing.

\section{Data collection}

Data were collected in three ways with the purpose of understanding how eMRs were used in patient encounters and the practitioners' and patients' perceptions and experiences.

\section{Structured observations}

Over 8 weeks excluding weekends, two researchers (LB, RJ) observed practitioners using eMRs in a variety of encounters with patients, including: admission assessments, allied health assessments, nursing handovers, inpatient and outpatient medical encounters, goalplanning and care-planning meetings. In up to five observations of each encounter type, the observer recorded: number and type of practitioners present, time spent with the patient, general topics discussed, when information was accessed and PCC strategies used during the encounter. No patient data were recorded. Unlike the Work Observation Method by Activity Timing ${ }^{17}$ designed to assess efficiency and safety, the tool developed in the present study primarily captured information regarding how PCC is maintained when practitioners use electronic devices. Raw data were entered into an Excel (Microsoft, Redmond, WA, USA) spreadsheet.

\section{Patient survey}

After an observation, a brief survey was completed face-to-face with participating patients about their interactions with practitioners in an eMR environment. The survey was based on the Picker Patient Experience Questionnaire, which assesses respect for patients' values, preferences and expressed needs; care coordination and integration; information, 
communication and education; physical comfort; emotional support and alleviation of fear and anxiety; involvement of family and friends; continuity and transition; and access to care after discharge ${ }^{18}$. Survey responses were recorded in an Excel (Microsoft) spreadsheet for analysis. All study data were de-identified and stored securely.

\section{Focus groups}

Participating practitioners were invited to attend a focus group conducted after the period of direct observation. Two researchers (MF, LB) facilitated the groups using a discussion guide based on key topics: eMR work and impact on patient interactions and work processes, including team communication and coordination; challenges and opportunities for PCC; and compatibility of eMRs with PCC in the complex long-term care setting (Appendix 1). Discussions were audio-recorded and transcribed.

\section{Data analysis}

Descriptive statistics and qualitative analysis were conducted on the observations data. Descriptive statistics were used to analyse the patient feedback survey. Focus group transcripts were subjected to deductive and inductive thematic analysis.

\section{RESULTS}

The results report on 50 observations totalling 17.5 hours, 43 patient surveys, and nine focus groups with 53 practitioners ( 3 medical, 37 nursing, 13 allied health practitioners). Table 1 describes clinical participants. Three practitioners (4\%) declined to participate, as did one inpatient.

(Table 1 about here) 


\section{Observed encounters}

Table 2 presents a summary of the observed encounters. Most were nursing inpatient handovers and medical outpatient consultations. The observed encounters between outpatients and doctors suggest that eMRs worked well in this office setting.

(Table 2 about here)

\section{Patient surveys}

Table 3 summarises patients' survey responses. Almost all patients held positive opinions on the value of the eMR. A small majority had no family or friends present during the observed encounter, but the rest found their family and friends were included and respected. One patient reported that need for information regarding ongoing care treatment and services was not adequately met. A small majority felt that advice about post-discharge access to services was adequate. A minority reported that practitioners had not shared information with them from their electronic record.

(Table 3 about here)

\section{Focus groups}

The focus group analysis yielded three descriptive themes relating to the study aims. Quotes from participants are coded as follows: medical participant (MP), allied health participant (AHP), nurse participant (NP).

\section{Functionality of the technology}

Theme one comprises what can or cannot be accomplished with eMR compared to paperbased management of safety and quality, timely information retrieval and decision-making, 
patient monitoring, and efficiency. The functionality of the eMR has implications for interdisciplinary team-based planning including unintended consequences, both positive and negative:

Simultaneous access by multiple practitioners was beneficial because 'you don't need to wait (to document care)' (NP), and records were accessible 'from different parts of the hospital' (MP). Patients also noticed the value of quick access to their electronic records vs. paperbased records. Some believed the eMR was safer because their information was more secure and readable, while a minority expressed concern about their information being accessible to staff not involved in their care. Likewise, some practitioners agreed it was easy to overinform, and that 'everyone is going to read it' (AHP).

Legibility was thought to improve multidisciplinary communication and encourage entries to be read 'a bit more thoroughly than when it was just scrawl on paper' (NP). Automatic alerts of clinical changes requiring prompt attention also enabled efficiencies:

It's notifying you and the doctors are being notified and you're not running around to find a doctor to tell them something is going on (NP).

Nonetheless, information retrieval could be complicated and drawn-out, because 'people are documenting it in so many different places' (AHP)

For nursing participants in particular, the generic eMR lacked versatility to cater for specialised rehabilitation, and was thought by some to be 'superficial...scraping the surfaces of things that we do' (NP), thus under-representing the scope, primacy and intensity of activities such as 'trend(ing) catheter changes, the skin, the things that are relevant for rehab' 
(NP), or to see on one page patients' progress 'from wheelchair to Canadian crutches to walking with a cane' (NP). Despite practitioners' concerns, all but one patient felt their information needs were met regarding ongoing care and services.

Paradoxically for nurses, the straightforward one-chart, paper-based care plan had been superseded by multiple paperless care plans for 'the skin...falls...wounds...smoking' (NP), adding complexity and time to the work of planning and recording care. Now 'it feels like we've tripled the documentation' (NP). In some cases, the level of necessary adaptation triggered the development of ad hoc, inconsistent solutions:

If you're not sure you've done it properly here, you go and do it somewhere else as well, to make sure that you've tried to cover it. And then you're verbally making sure you hand that over as well, so if people don't see it written, hopefully they've heard it (NP).

Adaptation was an undercurrent in this theme as digitalisation had streamlined some aspects of practice while adding unexpected complexity. As the potentially highest users of eMRs, nurses faced substantial daily challenges to accommodate their specialised work into the generic model, and to keep other members of the clinical team informed.

\section{Usability of the technology}

Theme two reveals how practitioners used eMRs in daily tasks, workflows and patient encounters, and the consequences for teamwork and practice generally. Unsurprisingly, eMRs alter workflows and reduce opportunities for informal multidisciplinary interaction.

Although auditing processes were now easier, nursing workflows were constrained by documentation complexity because 'you can't stop in between ... and document every little 
thing' (NP). Due to 'lots of black spots' (NP) in some clinical areas, the automatic documentation of clinical data such as blood pressure failed, and had to be re-done, so nurses usually also hand-recorded these observations to transcribe later: I just write them down...so I just save myself a whole step (NP). New tasks and pressures seemed to offset the benefits of the paperless system:

It's time-consuming waiting for the computers to open up, get to the patient that you want, there's several different pages that you might need to record (on)...your patient's buzzing, you... $\log$ off, go and do what you've got to do with your patient, then...come back and log on and try to get to the page again (NP).

Deferred documentation became an efficiency measure, strengthened by written checklists for tailored care needs noted during handover:

I can't remember, unless I go to my notes, and my piece of paper with the handover, and I make little boxes, and if I do those tasks I tick them, because you get interrupted (NP).

The time dedicated to completing documentation was perceived to disrupt teamwork and responsiveness to patients:

Everyone's concerned, making sure they get the paperwork done in time...(and patients) are waiting for their own nurse allocated to that area to (respond) (NP).

Decentralised documentation diminished valuable interdisciplinary information-sharing, because: ...[W]e've lost that bit of informal communication we used to get by sitting at the 
nurse's station, where the charts were. And you just overhear conversation and ...you might intervene sooner. Or people approach you because you're there (AHP).

This theme reveals how eMR procedures can re-orient workflows, whereby documentation might be deferred rather than contemporaneous to conserve efficiency. Notably, informal relationships and communications around paper-based documentation may be disrupted by the transfer to eMR.

\section{Meaning and value for person-centred care}

Theme 3 focuses on participants' perspectives on person-centredness in the eMR environment, and reveals the impact on practitioner-patient interactions, patients' preferences, and continuity and coordination of care.

The focus groups highlighted nurses' concerns that interactions with inpatients had been displaced by time-intensive eMR documentation. In particular, person-centredness seemed elusive, undermining the quality of the practitioner-patient relationship:

[C]onversation is gone, because I have to spend an hour to two just on documentation that used to take me half an hour (NP).

The perception was that practitioners generally now resorted to patients' records for information more readily than to patients themselves:

[Instead of] asking the patient the questions...now, everybody's gravitating towards this computer to have a look at what's been written last (NP). 
Nurses were also concerned about the intrusion of technology into patient encounters, and what this signified for their patients, because 'you're looking at the screen instead of looking at your patient' (NP). They opted for discretionary use of eMRs to maintain personcentredness:

When [patients] are really upset because they can't walk, I have to try and deal with this, so I just ignore the computer. Because you're a nurse, you're there for the patients, you're not there for the computer (NP).

The generic eMR seemed inconsistent with person-centredness in ways that were not easily adaptable. For example, while patients formerly held a copy of their goal plan as it puts the patient in control of their own rehab and goals' (AHP), but this plan could not now be directly printed from eMR documentation for patients before planning meetings.

In the inpatient setting, using eMRs had subtly changed how practitioners communicated with one another and with patients. Some nurse participants noticed that eMRs reinforced the power differential and distance between practitioners and patients:

Our patients are in wheelchairs, so they're always in the seated position, so even when the doctors are talking to the patients, if they've got the [workstations] up at the standing height they're talking down to the patient...it's always like we're over them, rather than sitting level with them (NP).

In particular, findings from the observed encounters showed a similar tension between the eMR and PCC. In the nursing handovers, most (66\%) used eMRs to conduct safety checks, focusing on checklists rather than patients. In contrast, most medical outpatient encounters 
(71\%) involved patient assessment, requiring interaction with patients. Despite this, almost all $(95 \%)$ patients agreed or strongly agreed that they were treated with respect, well-informed and involved in decisions about their care.

This theme reveals some practitioners' uneasiness about technology taking precedence and imposing extra documentation. This dilutes practitioner-patient relationships and tailored care in long-term rehabilitation. Nurses in particular resist the intrusion of eMR into the relational basis of PCC, and give precedence to their patients' specialised needs.

\section{DISCUSSION}

This research highlights the paradox of technology intended to streamline practice that often also intensifies and disrupts work for frontline rehabilitation practitioners. This is consistent with sociotechnical system theory, which recognises successful change as a process requiring due consideration of people, machines and context ${ }^{19}$. The present research thus reports a work in progress, as the eMR technology continues to be refined over time, in context and by people. Adaptation was imperative to preserve quality of care alongside eMR functionality in a complex multidisciplinary specialty that straddles health sectors, involves long-term therapeutic relationships, and requires coordination of multiple services. The types of encounter and practitioners observed were comprehensive, and revealed the challenges of reconciling a standardised eMR system and the highly tailored approach to spinal cord injury rehabilitation. Nonetheless, practitioners' commitment to person-centredness was apparent in the mean time spent with patients (21 minutes). This is noteworthy since the presence of technology is reported to reduce practitioners' direct time for patients ${ }^{2,20,21}$. The observed encounters between outpatients and doctors suggest that eMRs worked well in the office setting. 
Nurses" "failure" to begin patient encounters with conversation illustrates their respect for patients' preference to remain undisturbed during early morning bedside handovers. Patients' positive perceptions are encouraging, and consistent with previous findings regarding patient satisfaction with nursing time ${ }^{22}$. It is likely that a "neither" response in the patient survey indicated a non-applicable question since family or friends may have been absent, and postdischarge services may not have been discussed. Eight inpatients disagreed or strongly disagreed that information from their eMR had been shared with them, but limited bedside space in rehabilitation would make it difficult to manoeuvre a mobile workstation within reading distance for the patient during nursing handovers.

In the absence of an instrument to measure rehabilitation patient experience over time and across services and sectors, a general survey was used which may not have fully captured patients' experience. Further, these results reflect the views of a convenience sample, and potential bias limits transferability to other populations. However, they provide qualitative insights into an important topic.

Although eMR use brought challenges as well as benefits, it seems likely that eMR technology will remain. Others ${ }^{23}$ recommend perseverance during an extended settling-in period, and the present results suggest that commitment to care will in time conquer the challenges of practising in a digital rehabilitation environment.

\section{CONCLUSION}

The use of eMR impacted practitioners in different ways, depending on the task-orientation of their discipline, with nurses experiencing most pressure. Tensions between eMR and personcentredness in day-to-day practice were resolved in-context through the less visible work of frontline practitioners' emergent discretionary actions, highlighting their persistence, 
adaptability and commitment to person-centredness in the digital environment. Unexpected challenges of technological change, such as loss of informal communications, may resolve gradually as people and contextual factors influence and improve eMR use.

\section{Acknowledgements}

This research was funded in-kind by The Hopkins Centre and the Division of Rehabilitation at Metro South Health Service, Queensland Health. We acknowledge the participants for their valuable contribution to this research. 


\section{REFERENCES}

1. World Health Organization. Service delivery and safety: WHO global strategy on peoplecentred and integrated health services. Interim Report. Geneva: World Health Organization, 2015.

2. Asan O, Smith P, Montague E. More screen time, less face time - implications for EHR design. J Eval Clin Pract 2014;20(6):896-901.

3. Øvretveit J, Scott T, Rundall T, Shortell S, Brommels M. Improving quality through effective implementation of information technology in healthcare. Int J Qual Health Care 2007;19(5):259-66.

4. Levy R, Pantanowitz L, Cloutier D, Provencher J, McGirr J, Stebbins J, et al. Development of electronic medical record charting for hospital-based transfusion and apheresis medicine services: Early adoption perspectives. J Pathol Inform 2010;1:8.

5. Bates D. Getting in Step: Electronic Health Records and their Role in Care Coordination. J Gen Intern Med 2010;25(3):174-6.

6. Buntin M, Burke M, Hoaglin M, Blumenthal D. The Benefits Of Health Information Technology: A Review Of The Recent Literature Shows Predominantly Positive Results. Health Affair 2011;30(3):464-71.

7. Jarvis B, Johnson T, Butler P, O'Shaughnessy K, Fullam F, Tran L, et al. Assessing the impact of electronic health records as an enabler of hospital quality and patient satisfaction. Acad Med 2013;88(10):1471-7.

8. Garvey T, Evensen A. Increased patient communication using a process supplementing an electronic medical record. WMJ 2015;114(1):21-5.

9. Woods S, Schwartz E, Tuepker A, Press N, Nazi K, Turvey C, et al. Patient experiences with full electronic access to health records and clinical notes through the My HealtheVet Personal Health Record Pilot: qualitative study. J Med Internet Res 2013;15(3):e65. 
10. Payne TH, Corley S, Cullen TA, Gandhi TK, Harrington L, Kuperman GJ, et al. Report of the AMIA EHR 2020 Task Force on the Status and Future Direction of EHRs. J Am Med Inform Assoc 2015;0:1-11.

11. Raddock M, Lawson P, Smith S. Transitions, realignments, and focus shifts: possibilities for biopsychosocial care in the electronic health record era. Int J Psychiatry Med 2014;47(4):299-308.

12. Varpio L, Rashotte J, Day K, King J, Kuziemsky C, Parush A. The EHR and building the patient's story: A qualitative investigation of how EHR use obstructs a vital clinical activity. Int J Med Inform 2015;84(12):1019-28.

13. Darr A, Harrison M, Shakked L, Shalom N. Physicians' and nurses' reactions to electronic medical records: Managerial and occupational implications. J Health Organ Manag 2003;17(5):349-59.

14. Friedman A, Crosson JC, Howard J, Clark EC, Pellerano M, Crabtree BF, et al. A Typology of Electronic Health Record Workarounds in Small-to-Medium Size Primary Care Practices. J Am Med Inform Assoc 2014;21:e78-e83.

15. Crampton N, Reis S, Shachak A. Computers in the clinical encounter: a scoping review and thematic analysis. J Am Med Inform Assoc 2016;pii: ocv178.

16. Burton-Jones A, Grange C. From Use to Effective Use: A Representation Theory Perspective. Inform Syst Res 2013;24(3):632-58.

17. Westbrook J, Ampt A, Kearney L, Rob M. All in a day's work: an observational study to quantify how and with whom doctors on hospital wards spend their time. Med J Aust 2008;188(9):506-9.

18. Jenkinson C, Coulter A, Bruster S. The Picker Patient Experience Questionnaire: development and validation using data from in-patient surveys in five countries. Int $J$ Qual Health Care 2002;14(5):353-8. 
19. Baxter G, Sommerville I. Socio-technical systems: From design methods to systems engineering. Interact Comput 2011;23(1):4-17.

20. Block L, Habicht R, Wu A, Desai S, Wang K, Silva K, et al. In the Wake of the 2003 and 2011 Duty Hours Regulations, How Do Internal Medicine Interns Spend Their Time? J Gen Intern Med 2013;28(8):1042-7.

21. Payne T, Corley S, Cullen T, Gandhi T, Harrington L, Kuperman G, et al. Report of the AMIA EHR-2020 Task Force on the status and future direction of EHRs. J Am Med Inform Assoc 2015;22(5):1102-10.

22. Higgins L, Shovel J, Bilderback A, Lorenz H, Martin S, Rogers D, et al. Hospital Nurses' Work Activity in a Technology-Rich Environment: A Triangulated Quality Improvement Assessment. J Nurs Care Qual 2017;32(3):208-17.

23. Snowden A, Kolb H. Two years of unintended consequences: introducing an electronic health record system in a hospice in Scotland. J Clin Nurs 2017;26(9-10):1414-27. 
Table 1. Focus group participant demographics $(n=53)$

\begin{tabular}{lcc}
\hline Characteristic & $n$ & $(\%)$ \\
\hline Female & 48 & $(91)$ \\
Professional field & & \\
Medicine & 3 & $(5.5)$ \\
Nursing & 37 & $(70)$ \\
Allied Health & 13 & $(24.5)$
\end{tabular}

Time since graduation (range $1-37$ years, mean $=13$ years)

$\square 2$ years $\quad 7$

$3-5$ years

$>5$ years 34

$\begin{array}{ll}\text { Postgraduate training } & 7\end{array}$

Time in current position ${ }^{\mathrm{A}}$ (range 2 months-30yrs, mean $=$

$7.5 \mathrm{yrs})$

$<1$ year

$1-5$ years

22

$>5$ years

23

Current working status

Full-time $\quad 30$

Part-time

$23 \quad(43)$

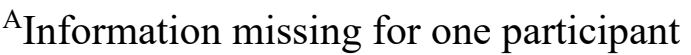


Table 2. Summary of observed encounters

\begin{tabular}{|c|c|}
\hline Type of encounter $(n=50)$ & $n$ \\
\hline Nursing handovers & 18 \\
\hline Medical outpatient consultations & 17 \\
\hline Goal-planning meetings & 7 \\
\hline Bedside medical rounds & 5 \\
\hline Admission assessments & 2 \\
\hline Nutritional assessment & 1 \\
\hline Length of encounters (mean (range)) & $21(1-66)$ minutes \\
\hline Person-centredness during encounters & Proportion of encounters $(\%)$ \\
\hline \multicolumn{2}{|l|}{ Doctor-outpatient } \\
\hline Encounter commenced with conversation & $94 \%$ \\
\hline Computer ignored during encounter & $88 \%$ \\
\hline Screen shared with patient & $59 \%$ \\
\hline Screen time used to talk with patient & $71 \%$ \\
\hline \multicolumn{2}{|l|}{ Doctor-inpatient } \\
\hline Encounter commenced with conversation & $100 \%$ \\
\hline \multicolumn{2}{|l|}{ Nurse-inpatient } \\
\hline Encounter commenced with conversation & $11 \%$ \\
\hline
\end{tabular}


Table 3. Summary of patient survey results $(n=43)$

\begin{tabular}{|c|c|c|c|}
\hline & $\begin{array}{l}\text { Agree or } \\
\text { strongly } \\
\text { agree }^{\mathrm{A}}\end{array}$ & Neither & $\begin{array}{l}\text { Disagree or } \\
\text { strongly } \\
\text { disagree }^{\mathrm{A}}\end{array}$ \\
\hline During this visit... & & & \\
\hline My needs were treated respectfully & $42(98 \%)$ & 1 & 0 \\
\hline I was involved in decision-making & $41(95 \%)$ & 1 & $1(2 \%)$ \\
\hline My care was well organised & $40(93 \%)$ & 3 & 0 \\
\hline The clinical team communicated well & $38(88 \%)$ & 5 & 0 \\
\hline I was fully informed on what is important to me & $41(95 \%)$ & 2 & 0 \\
\hline Clinicians shared information with me from eMR & $30(70 \%)$ & 4 & $9(21 \%)$ \\
\hline Clinicians addressed me personally & $42(98 \%)$ & 0 & $1(2 \%)$ \\
\hline My privacy needs were met & $42(98 \%)$ & 1 & 0 \\
\hline I was physically comfortable & $43(100 \%)$ & 0 & 0 \\
\hline My emotional needs were met & $37(86 \%)$ & 6 & 0 \\
\hline My family/friends were respected & $18(42 \%)$ & 25 & 0 \\
\hline My family/friends' needs were met & $19(44 \%)$ & 24 & 0 \\
\hline Ongoing treatment was explained & $34(79 \%)$ & 8 & $1(2 \%)$ \\
\hline I understood ongoing treatment & $42(98 \%)$ & 1 & 0 \\
\hline I received clear advice on service access after & $25(58 \%)$ & 18 & 0 \\
\hline
\end{tabular}

${ }^{\text {A}}$ Percentages rounded to the nearest whole figure. 


\section{APPENDIX 1. Focus group discussion guide}

\section{Overall experiences witheMR}

a) Preparation, expectations regarding change.

b) Operational challenges.

c) Benefits.

\section{Structure and functions of eMR}

a) What eMR can or cannot accomplish $v$. paper-based approach.

b) How problems are being resolved.

c) How eMR supports safety and quality (e.g. timeliness, decision-making, monitoring).

d) How eMR supports interdisciplinary teamwork.

e) Any unintended consequences, positive or negative.

\section{Usability of eMR}

a) How well eMR fits with clinical tasks, workflow, routines.

b) How eMR is incorporated into patient encounters and processes.

c) How eMR use impacts on team processes, roles, responsibilities.

d) Impact on quality, safety, efficiency, time for person-centredness.

\section{4. eMR should support person-centredness}

a) How use of eMR impacts on interaction with patients, quality and style of patient encounters.

b) How adequately eMR supports patient-specific instructions, needs, preferences, values.

c) Use of eMR to engage with patients or families. 
d) How eMR supports continuity, coordination and communication across providers and services.

\section{Any additional comments}

